Biochimica et Biophysica Acta, 397 (1975) 510-518

(C) Elsevier Scientific Publishing Company, Amsterdam - Printed in The Netherlands

BBA 67564

\title{
A PHYSICAL EXPLANATION OF THE EPR SPECTRUM OBSERVED DURING CATALYSIS BY ENZYMES UTILIZING COENZYME $B_{12}$
}

\author{
K.L. SCHEPLER, W.R. DUNHAM, R.H. SANDS, J.A. FEE and R.H. ABELES
}

Biophysics Research Division, Institute of Science and Technology, University of Michigan, Ann Arbor, Mich. 48105 and Graduate Department of Biochemistry, Brandeis University, Waltham, Mass. 02154 (U.S.A.)

(Received March 11th, 1975)

\section{Summary}

We have proposed that the "doublet" EPR spectra observed during catalysis by a number of coenzyme $B_{12}$-requiring enzymes arises from a weak electrostatic exchange interaction between an organic free radical and low spin $\mathrm{Co}(\mathrm{II}), \mathrm{B}_{12 \mathrm{r}}$. By varying the magnitude of the exchange coupling we have quite accurately simulated the published EPR spectra from the enzyme systems: diol dehydrase, glycerol dehydrase, ribonucleotide reductase, and ethanolamine ammonia lyase. A dipolar model was shown to be incompatible with the observed properties of these systems.

\section{Introduction}

Several enzyme systems requiring the vitamin $B_{12}$ coenzyme have been shown to exhibit unusual electron paramagnetic resonance (EPR) spectra during the course of catalysis. The observed spectra are substantially similar in all reported instances, and are characterized by a broad resonance near $g=2.3$ and by a rather narrow doublet disposed around $g=2$. Information relevant to the various enzyme systems was gathered from the published literature [1-8] and is assembled and referenced in Table I.

In every system studied the appearance of the characteristic EPR spectrum occurs at a rate which is faster than or comparable to the turnover rate of the enzyme. In the case of diol dehydrase from Aerobacter aerogenes $[9,10]$ it has been shown that all of the coenzyme molecules participate in a hydrogen exchange or transfer reaction with the substrate during catalysis and therefore all the coenzyme participates in the reaction. Since, in general, the integrated intensity of the EPR apparen $i$ substance, relative to a standard solution of $\mathrm{Cu}^{2+}$, accounts for a substantial fraction of the total coenzyme present it would 


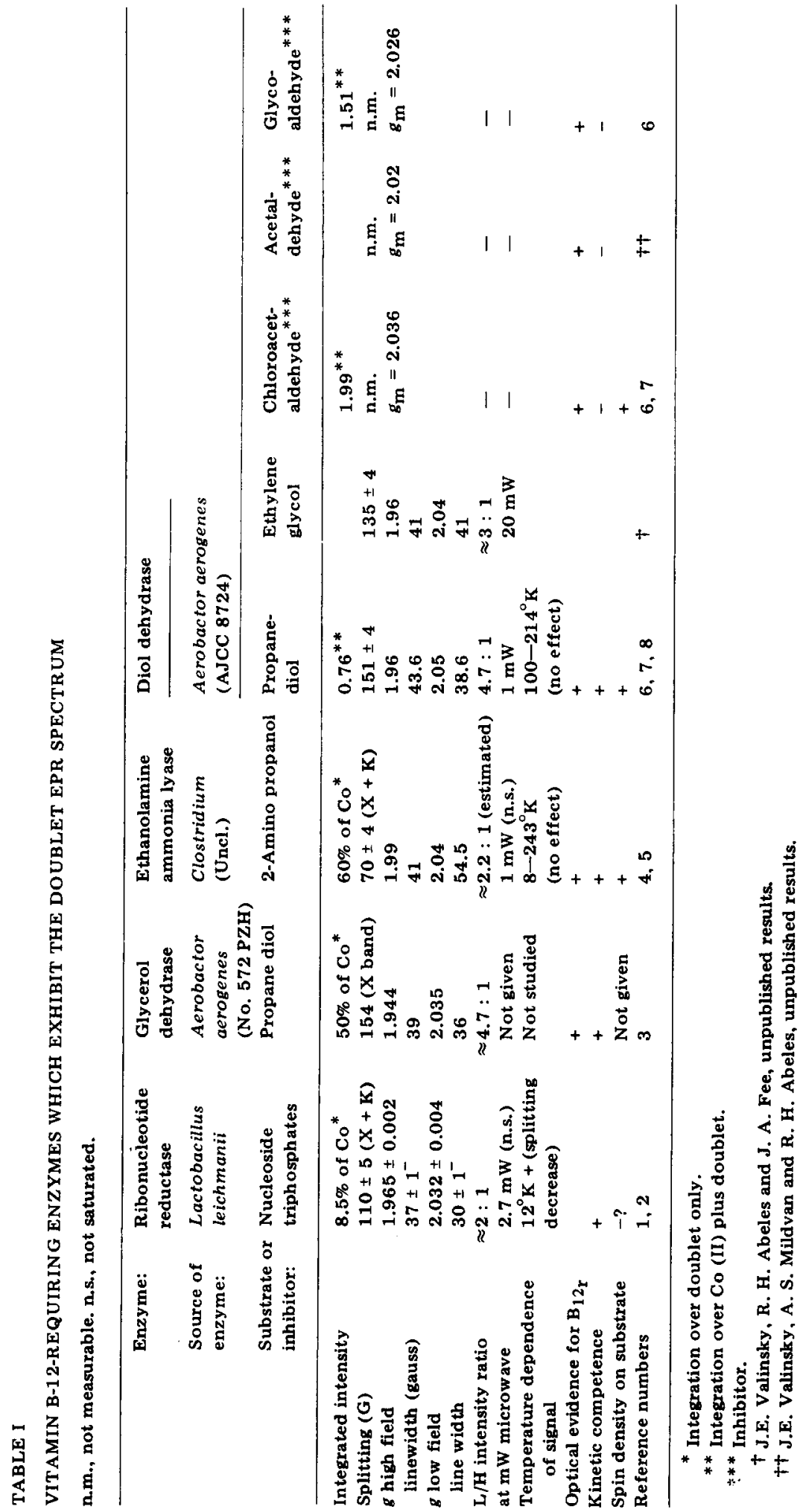


appear that the EPR spectrum arises from a viable intermediate of the catalytic reaction. We will offer further substantiation of this general conclusion.

A number of observations suggest that the EPR spectrum in question derives from an organic free radical and low spin $\mathrm{Co}(\mathrm{II}), \mathrm{B}_{12 \mathrm{r}}$. The evidence for the formation of $B_{12 r}$ during catalysis derives from both optical spectra and EPR spectra. Thus, during catalysis the optical spectrum of these enzymatic systems consists, at least in part, of that characteristic of $B_{12}$. And, the EPR signal evident near $g=2.3$, has been generally associated with $B_{12 \times}$ which has its strongest absorption at this energy.

Evidence for the presence of an organic free radical comes from experiments in which isotopic substitutions, either replacing ${ }^{1} \mathrm{H}$ with ${ }^{2} \mathrm{H}$ or ${ }^{12} \mathrm{C}$ with ${ }^{13} \mathrm{C}$ have been made in the various substrates and the affect of this on the EPR spectrum observed. In the cases of diol dehydrase (ref. 8 and Valinsky, J.E., Fee, J.A. and Abeles, R.H., unpublished) and ethanolamine ammonia lyase [5] it has been shown that some spin density exists on certain atoms of the respective substrates.

However, complete deuteration of a substrate of ribonucleotide reductase (guanosine triphosphate) yielded no evidence of spin density on the C-2 proton of this substance. Further, neither the use of deoxy $\left[5^{\prime}, 5^{\prime}-{ }^{2} \mathrm{H}_{2}\right]$ adenosyl cobalamin in place of the normally protiated coenzyme nor reduced seleno lipoate in place of reduced lipoate as the electron donor failed to induce any modification of the observed EPR spectrum [2]. Therefore, in two cases it has been demonstrated that electron density resides on the substrate and in the ribonucleotide reductase system ${ }^{13} \mathrm{C}$ substitution in the ribose ring, particularly at the 2 and 3 positions, may yet yield evidence of spin density on substrate.

The above discussion suggests that the EPR spectrum of the intermediate arises from a substrate radical and low spin $\mathrm{Co}$ (II), $\mathrm{B}_{12 \mathrm{r}}$. A number of observations have indicated that the two apparent resonances around $g=2$ are associated with a single specie: (a) The energy difference between the two lines is independent of the Zeeman energy, (b) isotopic substitutions affect each line in a similar fashion, (c) saturation studies indicate that the two lines have similar relaxation properties which differ substantially from those of the $g \cong 2.3$ resonance. These observations strongly suggest that the observed spectrum arises from some form of spin-spin interaction. The questions to be answered are the nature of the spin-spin interaction and the identity of the interacting specie.

The purpose of this communication is to demonstrate that the high field portion of all EPR spectra of this type observed to date can be accurately computed using a simplified model in which spin dipolar interactions are substantially smaller than spin exchange interactions and the primary cause of the splitting is an electrostatic exchange interaction between an organic free radical and low spin $\mathrm{Co}(\mathrm{II})$.

\section{Theory and comparison with published experimental observations}

That the spectra are caused predominantly by an isotropic exchange interaction between the low spin $\mathrm{Co}$ (II) and a free radical will be obvious to anyone schooled in high resolution NMR. The spectra in question bear striking resemblances to those observed in what is known in proton NMR circles as $A B$ systems, that is, a system consisting of exchange-coupled spins having relatively 
similar "chemical shifts" or g-values [12]. The major clues that this is the cause of the splitting are: (a) the line intensities of the "doublet" are markedly different for all spectra studied even when the individual line shapes are highly symmetric, and (b) the individual line shapes are highly symmetric whenever the splitting is large. By the same token these same clues indicate that the spectra do not result from a dipole-dipole coupling between two spin specie. For a discussion of spectra resulting from dipole-dipole coupling the reader is referred to any text on NMR in the solid phase (e.g. ref. 13). This having been said, most of what is to follow is redundant; nevertheless, for reasons of continuity the physics will be repeated.

The system under investigation here is slightly more complicated than proton NMR because the Co(II) has an anisotropic $g$-tensor. That fact only complicates the mathematics and is superfluous to the important physics of the problem, therefore, we will begin by discussing the case of two interacting spin specie having isotropic $g$-tensors. The main features of the spectra will show themselves there, and the added complication of anisotropic $g$-tensors will be discussed last.

Let us write the Hamiltonian describing the system of two spin 1/2 electrons interacting with an isotropic exchange interaction and in an applied magnetic field as follows:

$\mathscr{K}=-g_{1} \beta \vec{S}_{1} \cdot \vec{H}-g_{2} \beta \vec{S}_{2} \cdot \vec{H}-2 J \vec{S}_{1} \cdot \vec{S}_{2}$

$\mathscr{X}=-\left(g_{1} \beta S_{1 z}+g_{2} \beta S_{2 z}\right) H-2 J \vec{S}_{1} \cdot \vec{S}_{2}$

The interaction $S_{1} \cdot S_{2}$ commutes with $S_{z}=S_{1 z}+S_{2 z}$. If we choose the product representation given by the spin wavefunctions $\alpha(1) \alpha(2), \alpha(1) \beta(2)$, $\beta(1) \alpha(2)$ and $\beta(1) \beta(2)$ (where $\alpha$ is the wavefunction for $m_{z}=+1 / 2$ and $\beta$ is the wavefunction for $\left.m_{\mathrm{z}}=-1 / 2\right)$ then the exchange interaction mix es the $\alpha(1) \beta(2)$ and $\beta(1) \alpha(2)$ states as stationary states. To obtain the new energy levels and stationary states one simply diagonalizes the matrix of energy eigenvalues. This has already been done for us in the NMR literature [14]. If a positive quantity $C$ and angle $\theta$ are defined by:

$C=1 / 2\left[\left(g_{2}-g_{1}\right)^{2} \beta^{2} H^{2}+4 J^{2}\right]^{1 / 2}$ and $\tan 2 \theta=\frac{2 J}{\left(g_{2}-g_{1}\right) \beta H}$

then the wavefunctions and energy levels for the coupled electron system are given as in Table II.

TABLE II

WAVE FUNCTION AND ENERGY LEVELS FOR TWO COUPLED ELECTRON SYSTEMS

\begin{tabular}{lll}
\hline$n$ & $\phi_{n}$ & $E_{n}$ \\
\hline 1 & $\alpha \alpha$ & $\frac{1}{2}\left(g_{1}+g_{2}\right) \beta H+\frac{1}{2} J$ \\
2 & $\cos \theta(\alpha \beta)+\sin \theta(\beta \alpha)$ & $-\frac{1}{2} J+C$ \\
3 & $-\sin \theta(\alpha \beta)+\cos \theta(\beta \alpha)$ & $-\frac{1}{2} J-C$ \\
4 & $\beta \beta$ & $-\frac{1}{2}\left(g_{1}+g_{2}\right) \beta H+\frac{1}{2} J$ \\
\hline
\end{tabular}




\begin{tabular}{lrl}
\hline Transition & Relative energy & Relative intensity \\
\hline $3 \rightarrow 1$ & $J+C$ & $\left(g_{1} \cos \theta-g_{2} \sin \theta\right)^{2}$ \\
$4 \rightarrow 2$ & $-J+C$ & $\left(g_{1} \cos \theta+g_{2} \sin \theta\right)^{2}$ \\
$2 \rightarrow 1$ & $J-C$ & $\left(g_{2} \cos \theta+g_{1} \sin \theta\right)^{2}$ \\
$4 \rightarrow 3$ & $-J-C$ & $\left(g_{2} \cos \theta-g_{1} \sin \theta\right)^{2}$
\end{tabular}

Because the spin system interacts with an oscillating microwave field along the $x$-axis, the interaction inducing transitions between states is given by $-g_{1} \beta S_{1 x} H_{x}-g_{2} \beta S_{2 x} H_{x}=-\beta\left(g_{1} S_{1 x}+g_{2} S_{2 x}\right) H_{x}$ and the major transitions induced are given by $\Delta S_{z}= \pm 1$ with relative intensities given by the square of the matrix elements as shown in Table III.

The spectrum will consist of four lines and have the general "stick-spectrum" appearance as shown in Fig. 1. The two lines on one side correspond to the radical "doublet". The ratio of the intensities of one of the middle lines to the outside line is given by:

$\frac{\left(g_{1} \cos \theta+g_{2} \sin \theta\right)^{2}}{\left(g_{1} \cos \theta-g_{2} \sin \theta\right)^{2}} \simeq \frac{1+\sin 2 \theta}{1-\sin 2 \theta}$ if $g_{1} \simeq g_{2}$

where $\theta$ is defined by Eqn 2. Let us calculate the expected ratio of these intensities by substituting approximate values for $J$ and $g_{1}$ and $g_{2}$ for a particular spectrum, e.g. that observed by Hamilton and Blakeley [1] for ribonucleotide reductase, shown in Fig. 2E. Notice that the separation of the "radical doublet" is a direct measure of $2 J(2 J \cong 110 \mathrm{~g})$ and $g_{1}=2.0, g_{2} \simeq 2.2$ so tan $2 \theta \simeq 110 \mathrm{~g} / 330 \mathrm{~g}=1 / 3$ and $\sin 2 \theta \simeq \tan 2 \theta \simeq 1 / 3$. Substituting these values in Eqn 3 , our predicted intensity ratio is $2: 1$, which is close to that observed. Clearly this precise agreement is accidental but, nevertheless, this points up the applicability of the simplified model.

Now that we understand the basic phenomenon responsible for these spectra we can expand our model to include the added complication caused by the fact that the $\mathrm{Co}$ (II) $g$ is a tensor and there is an anisotropic hyperfine interaction between the cobalt electron system and the cobalt nucleus. The

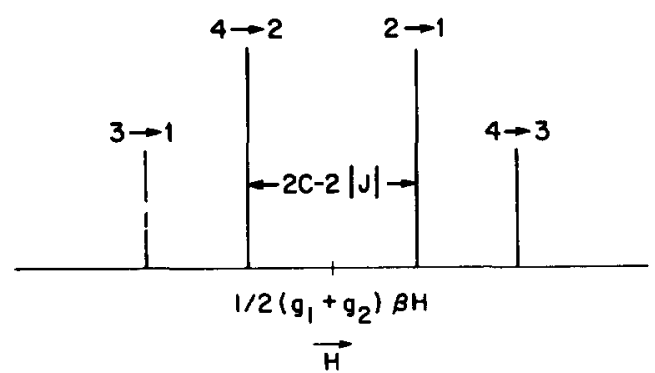

Fig. 1. Stick spectrum for classical AB spectrum. See text for explanation of symbols. (Taken from ref. 12, p. 122.) 


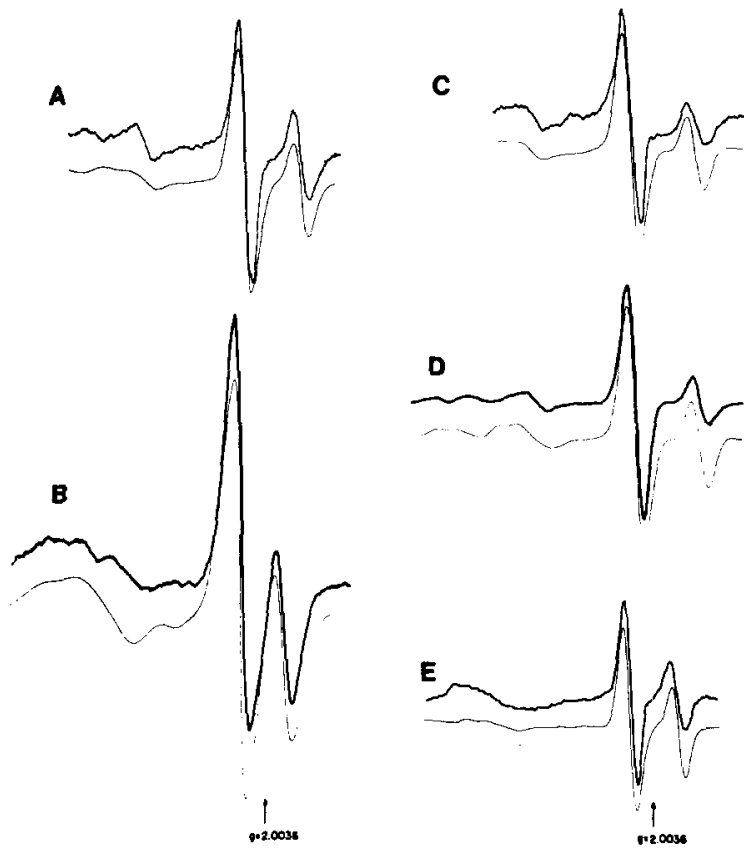

Fig. 2. Observed (upper) and computed (lower) EPR spectra of various enzyme-coenzyme $B_{12}$ substrate reaction mixtures. A, diol dehydrase plus ethylene glycol (Valinsky, J.E., Fee, J.A. and Abeles, R.H., unpublished); B, ethanolamine ammonia lyase plus L-2-aminopropanol [5]; C, diol dehydrase plus propane diol [3]; D, glycerol dehydrase plus propane diol; E, ribonucleotide reductase plus adenosine triphosphate [1]. Spectra were taken from the various references and were standardized photographically along the magnetic field. The computed spectrum was then normalized vertically to each spectrum. The parameters used in the computation are given in Table IV.

effective Hamiltonian is then

$\mathscr{K}=-\beta \vec{H}: \widetilde{g}_{2}: \vec{S}_{2}-g_{1} \vec{S}_{1} \cdot \vec{H}+\vec{I}_{2}: \widetilde{\widetilde{A}}_{2}: \vec{S}_{2}-2 \vec{S}_{1} \cdot \vec{S}_{2}$

We have treated these added terms by first-order perturbation theory assuming that the $A$-tensor principal axes were colinear with the $g$-tensor principal axes. A computer program was written which calculates a powder spectrum by taking a representative sampling of magnetic field orientations with respect to the g-tensor principal axis frame. The resulting stick-spectrum was then convolved with appropriate Gaussian lineshape functions to yield the final EPR spectrum.

Several representative spectra were calculated for the variety of interacting specie reported in the literature, and the comparisons with experimental spectra are shown in Fig. 2. The important fact to note here is that the cobalt parameters were nearly the same for all of these spectra (See Table IV). Because the intensity ratio of the radical "doublets" is sensitive to the cobalt parameters near perfect fits would result if these parameters were permitted to change slightly. We thought, however, that this presentation was more useful in demonstrating that the model of an exchange-coupled cobalt radical system explains all of the spectra to first order simply by varying the magnitude of the exchange coupling. 
TABLE IV

PAR AMETERS FOR THE SPECTR AL SIMULATIONS SHOWN IN Fig. 2

The Co(II) $A$-values used for all spectra were $A_{x}=35 \mathrm{MHz}, A_{y}=14 \mathrm{MHz}$ and $A_{z}=280 \mathrm{MHz}$. These values are consistent with those obtained for the $B_{12 r}$ system by Pilbrow and Winfield [11]. The $g$-tensor of the free radical, its linewidths, and the exchange coupling were taken to be isotropic. All linewidths are given in gauss $(\mathrm{G})$.

\begin{tabular}{|c|c|c|c|c|c|c|c|c|c|}
\hline $\begin{array}{l}\text { Enzyme } \\
\text { system } \\
\text { (shown in } \\
\text { Fig. 2) }\end{array}$ & $g_{x}$ & $\mathbf{g}_{y}$ & $\mathrm{~g}_{z}$ & $\left(\Delta H_{x}\right)_{1 / 2}$ & $\left(\Delta H_{y}\right)_{1 / 2}$ & $\left(\Delta H_{z}\right)_{1 / 2}$ & $\left(\Delta H_{\mathrm{rad}}\right)_{1 / 2}$ & $g_{\mathrm{rad}}$ & $\begin{array}{l}2 J \\
(\mathrm{MHz})\end{array}$ \\
\hline $\mathbf{A}$ & 2.2590 & 2.2284 & 1.9976 & 20 & 20 & 20 & 20 & 2.0049 & 378 \\
\hline B & 2.2833 & 2.2833 & 1.9982 & 40 & 40 & 20 & 30 & 2.0055 & 322 \\
\hline C & 2.2590 & 2.2360 & 1.9976 & 20 & 20 & 20 & 20 & 2.0049 & 434 \\
\hline D & 2.2523 & 2,2523 & 1.9976 & 20 & 20 & 20 & 20 & 2.0045 & 434 \\
\hline $\mathbf{E}$ & 2.2828 & 2.2828 & 1.9978 & 20 & 20 & 20 & 15 & 2.0051 & 322 \\
\hline
\end{tabular}

It should be emphasized that the assumptions made by previous authors that the "doublet" was from a single $S=1 / 2$ specie are substantiated by these spectral fits, and the integrations previously alluded to are correct.

The readers attention is called to the fact that not much magnetic dipoledipole interaction is permitted by these spectra; such computations (see below) resulted in broad anisotropic lineshapes over and above that introduced by the cobalt anisotropic $g$ - and $A$-tensors.

\section{Discussion}

The exchange-coupled model of a low-spin cobalt (II) and an organic radical adequately explains all spectra observed to date in these systems. In particular, it accounts for the intensity ratios of the radical "doublets" which had previously escaped rational explanation. The fact that agreement in the $g=2.3$ region is haphazard at best can be readily explained by the fact that some isolated $B_{12 r}$ material can contribute here as well. This is particularly true with ribonucleotide reductase [2] where the low-field fit is quite poor.

One of the interesting features of the exchange-coupled model is that there exists sufficient overlap between the cobaltous electron distribution and the organic radical unpaired electron distribution to provide the isotropic exchange interaction, yet the dipole-dipole interaction between the same two electron spin distributions must be no larger than the linewidths.

The magnetic dipole-dipole interaction between two distributed spin distributions is given to first order by the quantum-mechanical expectation value

$$
\left\langle\frac{\mu_{1_{z}} \mu_{2_{z}}}{r^{3}}\left(3 \cos ^{2} \theta-1\right)\right\rangle
$$

over the two spatial distributions, where $\theta$ is the angle between the applied external field and $\vec{r}$ is the radius vector connecting the two dipoles. Because the samples are all frozen aqueous solutions, all orientations of the complex with respect to the external field are to be weighted equally according to solid angle [13]. This weighted sum produces an anisotropic distribution. In order to first 
integrate over the spin distributions themselves and then sum over the random orientations of the complex with respect to the external field, it is convenient to define a coordinate system fixed to the complex and then to specify the orientation of both $\vec{r}$ and the applied field, $\vec{H}$, with respect to this same coordinate system. By means of the spherical harmonic addition theorem [14],

$\frac{3 \cos ^{2} \theta-1}{2}=\frac{3 \cos ^{2} \nu-1}{2} \cdot \frac{3 \cos ^{2}(B)-1}{2}+\sum_{\substack{m=-2 \\ m \neq 0}}^{2}\left(\frac{2}{5}\right)^{1 / 2} Y_{2}^{-m}(\nu, \phi) Y_{2}^{m}(\mathbb{A}, \phi)$

it is possible to specify the value of $3 \cos ^{2} \theta-1$ in terms of the tesseral harmonics $Y_{2}^{\mathrm{m}}(\nu, \phi)$ and $Y_{2}^{\mathrm{m}}(\Theta \Theta, \Phi)$ where $\nu$ and $\phi$ are the spherical polar coordinates of $\vec{r}$ with respect to the axis system fixed to the charge distribution and $(A)$ and $\Phi$ are the spherical polar coordinates of $\vec{H}$ with respect to the same axes. This then permits us to integrate over the various orientations of $\vec{r}$ specified by the spin distributions. Clearly one may obtain small values of $\left\langle\left(3 \cos ^{2} \nu-1\right) / r^{3}\right\rangle$ if either $r$ is large or $\left\langle 3 \cos ^{2} \nu-1\right\rangle$ is small; thus, there are two physical mechanisms whereby the dipolar interactions can be diminished.

We do not know the distance between the two interacting spin systems, but one of these is fixed near the center of the corrin ring. This atom, Co(II), must play a central role in the catalytic mechanism and therefore may be thought to be in close proximity to the substrate. If the electron spin resides solely on substrate and this coordinates to $\mathrm{Co}(\mathrm{II})$ via one of its $\mathrm{C}, \mathrm{N}$, or $\mathrm{O}$ atoms, then for the small substrates the value of $r$ must be $<5 \AA$, assuming a maximum separation of the two unpaired electrons.

Because the cobalt paramagnetism is partially delocalized onto the atoms of the corrin ring, we will assume that only $50 \%$ of the spin density resides in the $3 \mathrm{~d}$ orbitals of cobalt. This assumption together with the assumption of $r \approx 5 \AA$ between centers of the unpaired spin distributions yield a value of $\left\langle\mu\left(3 \cos ^{2} \nu-1\right) / r^{3}\right\rangle<30 \mathrm{G}$. The intrinsic radical linewidths employed in the computer fits of Fig. 2 (see Table IV) ranged from 15 to $30 \mathrm{G}$, which are consistent with the above.

If $r<5 \AA$ then the dipolar interaction must be attenuated by a mechanism associated with $\left\langle 3 \cos ^{2} \nu-1\right\rangle$. Therefore, let us examine what it means for $\left\langle 3 \cos ^{2} \nu-1\right\rangle$ to also be small, since that is what the observation of a small dipolar coupling would require. There exists a "magic angle", $\nu=54^{\circ} 53^{\prime}$, such that $3 \cos ^{2} \nu-1$ is exactly zero. This leads us immediately to one possible model for the complex which would yield a vanishingly small dipolar coupling; namely, if the radical were bound to the cobalt in such a way that it was free to rotate (all values of $\phi$ ) around a cobalt-substrate bond where the angle between the line joining the center of the cobalt and the center of the dominant unpaired spin distribution makes an angle on the order of $54 \pm 15^{\circ}$ with respect to the normal of the corrin plane. The rotation frequency must be fast compared to the anisotropy in the dipolar coupling so as to average all of the $\phi$ terms to zero as they appear in the spherical harmonic addition theorem.

The attenuation of the dipolar interaction by rapid rotation of the organic radical as described above seems unlikely because those enzymes which act on large substrates exhibit EPR spectra essentially identical to those which have 
smaller substrates. Indeed, any free rotation model would seem to be excluded by the observations $[2,5]$ that the EPR spectra are unchanged at temperatures near liquid helium where thermally induced motion would be greatly reduced.

There is still a third mechanism by which the observed dipolar linewidth can be reduced, namely, if the interaction is modulated by either $T_{1}$ relaxation or by the radical going on and off the cobalt. This latter modulation mechanism has been invoked by Leigh [15] to explain the EPR spectra in free radicals covalently bonded to protein molecules containing paramagnetic ions. We cannot use it here because there is no evidence to support the notion that the radical is going on and off the cobalt. The relatively short electron $T_{1}$ of cobalt can be used, however, to explain some slight reduction in the observed dipolar splitting and for all but the lowest temperatures this undoubtedly contributes.

In summary, we believe that the model proposed here to explain the spectra observed is consistent with all of the physical and chemical information available. Any future chemical model proposed to explain the mechanism for these reactions must provide for the observed isotropic exchange interaction and must not require mean distances between radical and cobalt spin distributions much less than $5 \AA$.

\section{Acknowledgements}

Supported by Research Grants from the National Institutes of Health, GM-12176 (R.H.S.), GM-21519 (J.A.F.), GM-12633 (R.H.A.) and Training Grant, GM-1355 (K.L.S.).

\section{References}

1 Hamilton, J.A. and Blakeley, R.L. (1969) Biochim. Biophys. Acta 184, 224-226

2 Hamilton, J.A., Tamao, Y., Blakeley, R.L. and Coffman, R.E. (1972) Biochemistry 11, 4696-4705

3 Cockle, S.A., Hill, H.A.O., Williams, R.J.P., Davies, S.P. and Foster, M.A. (1972) J. Am. Chem. Soc. 94, 275-277

4 Babior, B.M., Moss, T.H. and Gould, D.C. (1972) J. Biol. Chem. 247, 4389-4392

5 Babior, B.M., Moss, T.H., Orme-Johnson, W.H. and Beinert, H. (1974) J. Biol. Chem. 249, 45374544

6 Finley, T.H., Valinsky, J., Mildvan, A.S. and Abeles, R.H. (1973) J. Biol. Chem. 248, $1285-1290$

7 Valinsky, J.E., Abeles, R.H. and Mildvan, S.A. (1974) J. Biol. Chem. 249, 2751-2755

8 Valinsky, J.E., Abeles, R.H. and Fee, J.A. (1974) J. Am. Chem. Soc. 96, 4709-4710

9 Frey, P.A., Essenberg, M.K. and Abeles, R.H. (1967) J. Biol. Chem. 242, 5369-5377

10 Essenberg, M.K., Frey, P.A. and Abeles, R.H. (1971) J. Am. Chem. Soc. 93, 1242-1251

11 Pilbrow, J.R. and Winfield, M.E. (1973) Mol. Phys. 25, 1073-1092

12 Pople, A., Schneider, W.E. and Bernstein, H.J. (1959) High Resolution Nuclear Magnetic Resonance, pp. 119-123, McGraw Hill, New York

13 Hecht, H.G. (1967) Magnetic Resonance Spectroscopy, pp. 84-89, J. Wiley and Sons, New York

14 Margenau, H. and Murphy, G.M. (1956) The Mathematics of Physics and Chemistry, p. 113, Van Nostrand, New York

15 Leigh, J.S. (1970) J. Chem. Phys. 52, 2608-2612 